\title{
Prolonged retention of primary teeth and TMD from the archaeological sample in the Edo Japanese
}

\author{
Hisashi Fujita \\ Department of Bioanthropology, Niigata College of Nursing, Japan, 240 Shinnan, Joetsu, 943-0147 Niigata, Japan
}

Email address:

hfujita@niigata-cn.ac.jp (H. Fujita)

To cite this article:

Hisashi Fujita. Prolonged Retention of Primary Teeth and TMD from the Archaeological Sample in the Edo Japanese. European Journal of Preventive Medicine. Vol. 2, No. 6, 2014, pp. 110-113. doi: 10.11648/j.ejpm.20140206.16

\begin{abstract}
Prolonged retention of bilateral primary second molars was observed in ancient human skeletal remains excavated from temple ruins of the middle to late Edo period in Japan. Radiographs showed no sign of bilateral second premolars which appeared to be congenitally missing. Furthermore, there was inflammatory deformation of the left mandibular condyle, and pseudarthrosis at the articulation with the left mandibular fossa and a small hole on the articular surface were observed. The case of this study is a rare case in which prolonged retention of bilateral primary second molars was speculated to have resulted in malocclusion, which in turn might have led to temporomandibular disorder. In addition, this case is reported here because its information is thought to be applicable to clinical practice in modern dentistry.
\end{abstract}

Keywords: Retention of Primary Teeth, TMJ Arthritis, TMD, Archaeology, Edo Japanese

\section{Introduction}

Prolonged retention of primary teeth rarely poses clinical problems in modern people if these teeth are extracted and the eruptive paths of permanent teeth are normalized. In the field of physical anthropology, one sometimes encounters ancient human skeletal remains with over-retained primary teeth, however, the scientific report is very few [1]. There have been various reports on temporomandibular joint (TMJ) arthritis and temporomandibular disorder (TMD) [2,3]. However, my literature search on the PubMed database did not reveal any report on the relationship between TMD and over-retained primary teeth. A report in a Japanese journal has shown that when primary teeth were retained beyond the normal period, their extraction resulted in gradual improvement of TMD and in normal occlusion [4].

In paleopathology which handles ancient skeletal remains, no report has been published that simultaneously discussed prolonged retention of primary teeth, TMJ arthritis, and TMD. The material of the present study was human skeletal remains from the Edo period $\left(17-19^{\text {th }}\right.$ centuries) in Japan. The mandible showed signs of TMJ arthritis and TMD likely due to bilateral primary second molars. Although the material was an excavated archeological sample, the author will report here on this material because the implication of its findings is thought to be important in modern dental medicine.

\section{Case Description}

The material was ancient human skeletal remains excavated from the Suhgen temple site in Shinjuku-ku in Tokyo. It was thought to be from the middle to late Edo period, the latter $17^{\text {th }}$ to the $19^{\text {th }}$ century. The material was labeled No. 181, and Kajigayama et al. had assessed the material [5]. They estimated its age to be in the early part of early middle age using the methods of Scheuer and Black [6], of Broca [7], of Ubelaker [8], and of Meindle and Lovejoy [9]. They determined its sex to be female using the features of the os coxae, especially the angle of the greater sciatic notch. The author of the present study observed a bony imprint on the preauricular sulcus of the left and right iliac bones, and the individual was strongly speculated to have been a parous woman. This material is now housed in the Department of Anthropology, National Museum of Nature and Science, Tokyo.

Retention of primary molars was observed in the left and right mandible (Figure 1). Since first premolars were present anterior to them, the over-retained primary teeth were both believed to be primary second molars. Radiographs showed no formation of left or right second premolars, confirming that they were missing congenitally (Figures. 2a and 2b). It was speculated that bilateral primary second molars remained in adulthood because second premolars were missing 
congenitally. The coronal dimensions were mesiodistally $10.79 \mathrm{~mm}$ and buccolingually $9.88 \mathrm{~mm}$ for the right primary second molar and $11.16 \mathrm{~mm}$ and $9.94 \mathrm{~mm}$, respectively, for the left primary second molar. Other permanent mandibular teeth, including the third molars, were erupted bilaterally, and it is consistent with the individual being in the early part of early middle age. Bilateral primary molars had more severe occlusal attrition of the distal area than on the mesial area, and dentin was markedly exposed (Figure 1). The occlusal attrition of the mesial area was confined to near the proximal aspect adjacent to the first premolar.

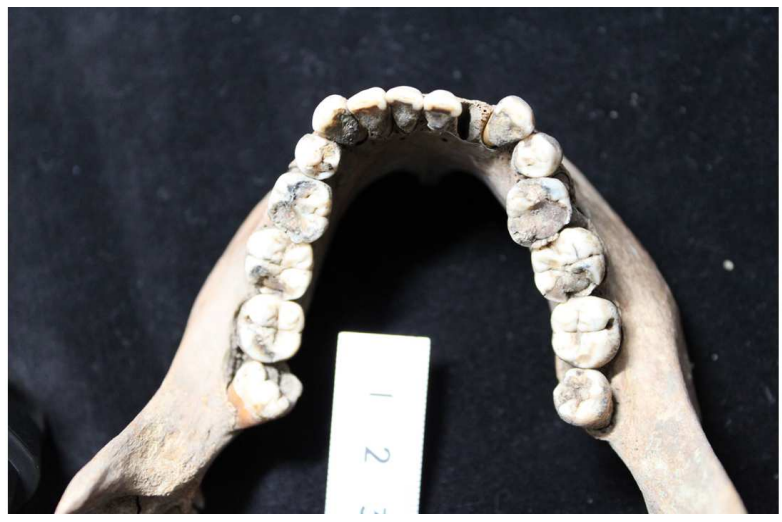

Figure 1. Persistence of both sides of second deciduous molars.
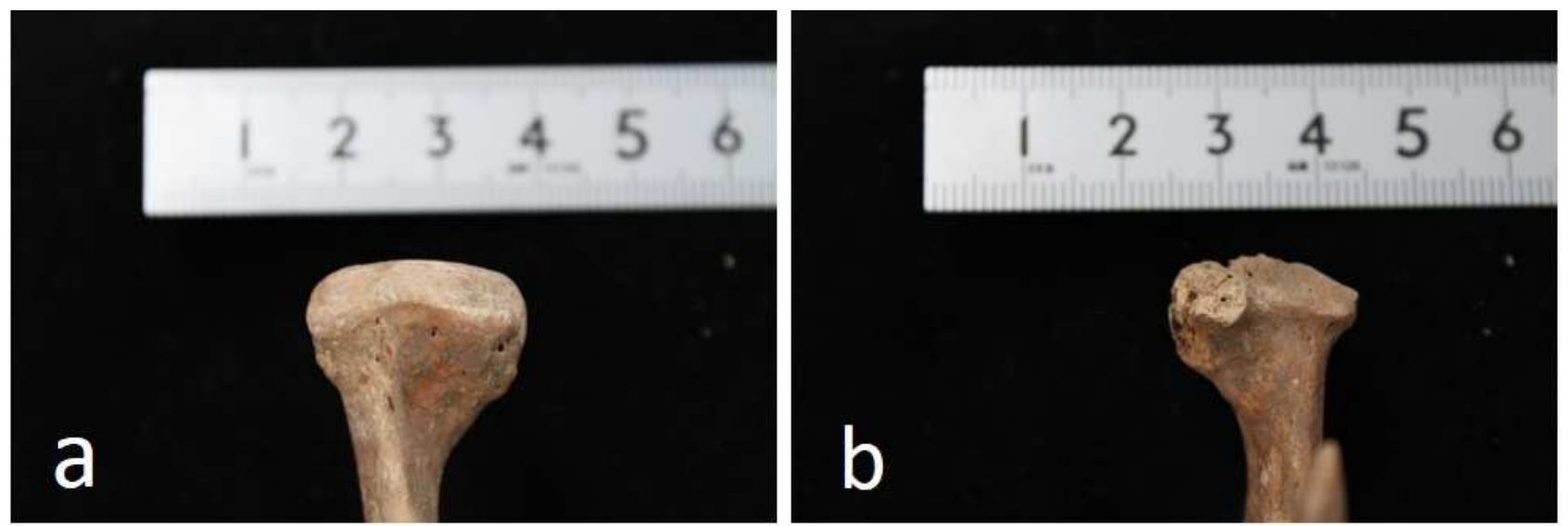

Figure 3. a: right mandibular condyle is normal. b: left mandibular condyle has caused the deformity by TMJ arthritis.
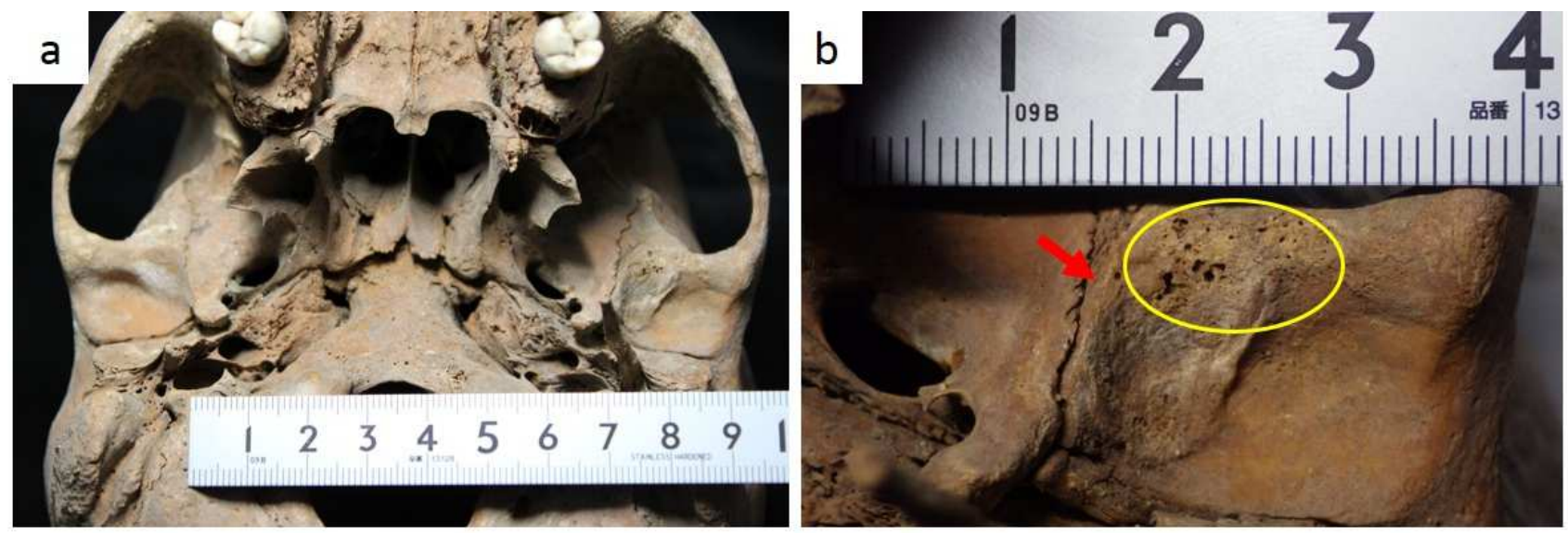

Figure 4. a: mandibular fossa of both sides. $b$ : left mandibular fossa forms the false joint (arrow) and porosity is recognized in frame area. 

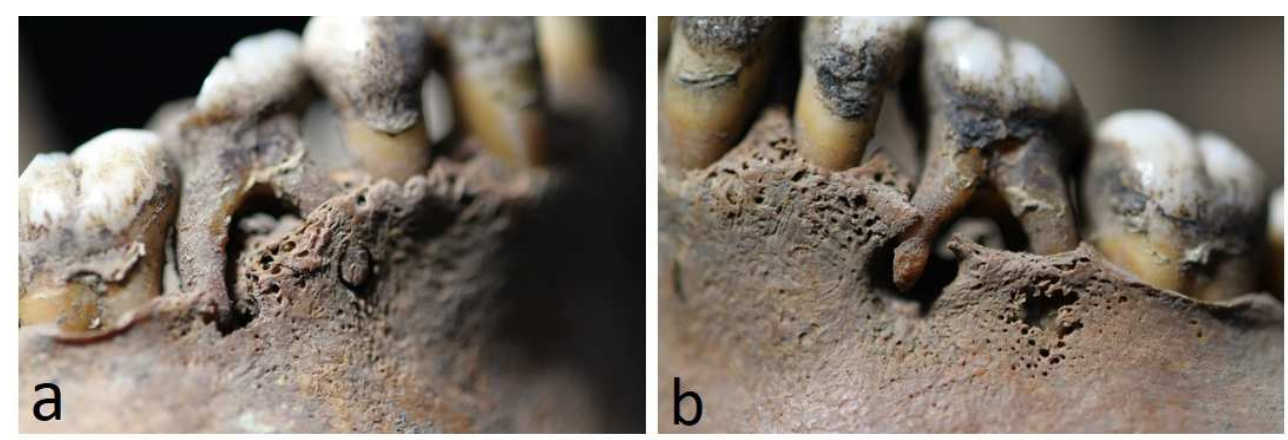

Figure 5. Periotitis found in the alveolar bone at right second deciduous molar (a) and left second deciduous molar (b).

\section{Discussion}

Sumiya reported that the prevalence of prolonged retention was $0.58 \%$ for primary second molars in both Japanese male and female aged 21-25 years [11]. Onizuka examined 151 teeth in 106 individuals with over-retained primary teeth who were aged $14-47$ years and reported that $63 \%$ had one overretained tooth and $32 \%$ had two over-retained teeth [12]. It should be noted that over-retained teeth occurred symmetrically in the left and right sides of the maxilla or mandible in 33 of 34 patients with two over-retained teeth, indicating a very high proportion of patients [12]. The most commonly over-retained tooth was the primary second molar at $52.3 \%$ [12]. In the present study, the individual had prolonged retention of two mandibular primary second molars with bilaterally symmetry. The occurrence rate was $0.19 \%$ based on simple calculation, indicating that such prolonged retention occurred in approximately 2 in 1000 adults. When one takes into account that the excavated human skeletal remains were of the Edo period, the individual in this study can be said to be a valuable case of over-retained teeth in ancient human skeletal remains.

Other interesting findings in the individual of this study were that inflammation had developed in the left mandibular condyle and deformation had clearly occurred. In the mandibular fossa, pseudarthrosis had formed and developed into TMJ arthritis. In the individual of the present study, occlusal attrition was observed in the distal area of the left and right first molars. In the right first molar, the occlusal surface gently sloped upward from the distal to the mesial direction until there was no attrition. One area of the left first molar showed severe attrition where its distal area contacted the left second molar. It is nearly impossible to reproduce an accurate premortem occlusion using a mandible and maxilla without soft tissue. However, there was likely abnormal occlusal force on the areas with attrition. When the maxillary dentition was examined, the teeth had normal positions in the left and right molar regions and there was slight attrition confined to the enamel. Attrition was confined to the lingual aspect for the left and right mandibular first molars. In the individual of the present study, the findings were strongly suggestive of malocclusion such as cusp-to-cusp occlusion. It is not known whether TMD occurred at a transition period from primary teeth to permanent teeth or after eruption of all permanent teeth. In any case, prolonged retention of primary second molars was thought to have somehow contributed to the development of TMD.

There are limitations in discussing TMD based on archeological materials, but there could be reports of new valuable cases of archeological materials. Much like the individual in the present study, modern-day individuals can have prolonged retention of primary teeth causing TMD and can have concurrent periodontal disease. Therefore, it is desirable to appropriately treat individuals with such conditions in modern clinical dental medicine.

\section{Acknowledgements}

The author thanks Dr. Y Mizoguchi of the Director of Department of Anthropology, National Museum of Nature and Science, Tokyo for their kind permission to study the material under his care. The author also wishes to express my gratitude to Dr. K Sakaue of the Department of Anthropology, National Museum of Nature and Science, who cooperated in taking the X-rays. Finally, the deep gratitude is dedicated to the following scientists who has written the excavation report; M Kajigayama, A Shirahase, E Ohtani, M Ozawa and Dr. H Baba of the Department of Anthropology, National Museum of Nature and Science, Tokyo.

\section{References}

[1] Fujita H, Suzuki T, Harihara S. (1997) Simultaneous Dental Anomalies (Polyanomalodontia) inMediaeval Japanese Skeletal Remains. Jpn J Oral Biol 39: 257-262.

[2] Murdoch B, Buchanan J, Cliff J. (2013) Temporomandibular joint replacement: a New Zealand perspective. Int J Oral Maxillofac Surg doi: 10.1016/j.ijom.2013.11.004.

[3] Singh S, Varghese D. (2013) Single puncture arthrocentesis of temporomandibular joint, introducing a novel device: A pilot study. Natl J Maxillofac Surg 4: 193-197.

[4] Horikawa Y, Okamoto K, Hasegawa S, et al. (1993) Two cases of children with craniomandibular disorder caused by the prolonged retation of the lateral $2^{\text {nd }}$ primary molar. Jpn J Ped Dent 31: 102-109. (in Japanese with English Summry)

[5] Kajigayama M, Shirahase A, Ohtani E, et al. (2005) Human skeletal Remains from Suhgen and Shouken temples sites. In: Suhgen and Shoken Temples Sites.Taisei Enjiniaringu ed, Tokyo: Meiji-jingu : 150-161. 
[6] Scheuer L, Black S. (2000) Development and ageing of Juvenile skeleton. In: Cox M, Mays S (eds.), Human osteology in archaeology and forensic science. London: Greenwich Medical Media, 9-21.

[7] Broca, P. (1879) Instructions relatives a I'etude anthropologique du systeme dentaire. Bull SOC Anthropol Paris 2:128-152.

[8] Ubelaker DH. (1989) Estimating age at death. In: Ubelaker DH, ed. Human skeletal Remains: Excavation, Analysis, Interpretation, 2nd edition. Washington, DC: Taraxacum: 6384.
[9] Meindle RS, Lovejoy CO. (1985) Ectocranial suture closure: A revised method for the determination of skeletal age at death based on the lateral-anterior sutures. Am J Phys Anthropol 68: 57-66.

[10] Rando C, Waldron T. (2012) TMJ Osteoarthritis: A New Approach to Diagnosis. Am J Phys Anthropol 148: 45-53.

[11] Sumiya Y. (1959) Statistic Study on Dental Anomalies in the Japanese. J Anthropol Soc Nippon 64: 215-233. (in Japanese)

[12] Onizuka Y. (1979) Statistical Observation on the Multiple Cases (106 Cases) of the Retained Deciduous Teeth. Kyushu Shika Gakkai Zasshi 33: 52-67. (in Japanese) 